Pharma, Fresenius, UCB, Grant/research support from: Celgene, Galapagos, Gilead, Lilly

DOI: 10.1136/annrheumdis-2021-eular.893

\section{POS0306 IMPACT OF MULTIMORBIDITY ON DISEASE MODIFYING ANTI-RHEUMATIC DRUG THERAPY IN EARLY RHEUMATOID ARTHRITIS: DATA FROM THE ESPOIR COHORT}

A. Beltai ${ }^{1}$, B. Combe ${ }^{1}$, A. Coffy' ${ }^{2}$ C. Gaujoux-Viala ${ }^{3}$, C. Lukas ${ }^{1}$, A. Saraux ${ }^{4}$, M. Dougados ${ }^{5}$, J. P. Daures ${ }^{2}$, C. Hua ${ }^{3}{ }^{1} \mathrm{CHU}$ Montpellier, Rheumatology, Montpellier, France; ${ }^{2}$ University Institute of Clinical Research, AESIO santé, Statistiques, Montpellier, France ${ }^{3} \mathrm{CHU}$ Nîmes, Rheumatology, Nîmes, France; ${ }^{4} \mathrm{CHU}$ Brest, Rheumatology, Brest, France; ${ }^{5} \mathrm{CHU}$ Cochin, Rheumatology, Paris, France

Background: Multimorbidity is frequent in rheumatoid arthritis (RA) and could interfere with the therapeutic response.

Objectives: The aim of this study was to evaluate multimorbidity in the French cohort of early arthritis (ESPOIR cohort) and its possible impact on the therapeutic response.

Methods: We included patients fulfilling 2010 ACR/EULAR criteria for RA. An adapted MultiMorbidity Index (aMMI) was developed [1]. Each patient was assigned scores of binary aMMI $(0=$ no comorbidity, $1=$ at least 1 comorbidity) and counted and weighted aMMI. The primary endpoint was achievement of Clinical Disease Activity Index (CDAI) low disease activity after initiation of a first disease-modifying anti-rheumatic drug (DMARD) according to the aMMI. Secondary endpoints were other disease activity scores and response criteria. We collected data from the visit preceding the first DMARD initiation (baseline visit) and the visit after at least 3 months of treatment (follow-up visit). The impact of aMMI on therapeutic maintenance at 1, 3,5 and 10 years was evaluated.

Results: Analyses involved 472 patients: 302 (64\%) had at least 1 comorbidity. Overall, $45.3 \%$ and $44.7 \%$ with binary aMMI $=0$ or 1 , respectively $(p=0.9)$, achieved CDAI low disease activity (Table 1 ). Similar results were found with counted and weighted aMMI. Use of other disease activity scores or response criteria did not show a significant impact of multimorbidity on the therapeutic response. Therapeutic maintenance was significantly better with binary $\mathrm{aMMI}=1$ than binary $\mathrm{aMMI}=0$. Increased counted $\mathrm{aMMI}$ was associated with increased probability of still being on the first initiated DMARD at each time point (Table 2).

Conclusion: In the ESPOIR cohort, therapeutic response to a first DMARD was not affected by multimorbidity but therapeutic maintenance was better in multimorbid patients.

REFERENCES:

[1] Radner H, Yoshida K, Mjaavatten MD, et al. Development of a multimorbidity index: Impact on quality of life using a rheumatoid arthritis cohort. Semin Arthritis Rheum 2015;45:167-73.

Table 1. Impact of aMMIs on CDAI, DAS28 and SDAl low disease activity (LDA) achievement at follow-up visit (univariate analyses)

\begin{tabular}{|c|c|c|c|c|c|c|c|c|c|}
\hline & & & & LDA ac & evemen & & & & \\
\hline & & & & & & & & & \\
\hline & Yes & No & $p$ & Yes & No & $p$ & Yes & No & $p$ \\
\hline Binary aMMI, n ( & & & & & & & & & \\
\hline 0 & $\begin{array}{c}77 \\
(45.3)\end{array}$ & $\begin{array}{c}93 \\
(54.7)\end{array}$ & $0.9^{*}$ & $\begin{array}{c}85 \\
(50.0)\end{array}$ & $\begin{array}{c}85 \\
(50.0)\end{array}$ & $0.2^{*}$ & $\begin{array}{c}80 \\
(47.1)\end{array}$ & $\begin{array}{c}90 \\
(52.9)\end{array}$ & $0.9^{*}$ \\
\hline 1 & $\begin{array}{c}135 \\
(44.7)\end{array}$ & $\begin{array}{c}167 \\
(55.3)\end{array}$ & & $\begin{array}{c}131 \\
(43.4)\end{array}$ & $\begin{array}{c}171 \\
(56.6)\end{array}$ & & $\begin{array}{c}141 \\
(46.7)\end{array}$ & $\begin{array}{c}161 \\
(53.3)\end{array}$ & \\
\hline $\begin{array}{c}\text { Counted aMMI, } \\
\text { mean (SD) }\end{array}$ & $\begin{array}{l}1.0 \\
(1.1)\end{array}$ & $\begin{array}{c}1.1 \\
(1.1)\end{array}$ & 0.7 & $\begin{array}{c}1.0 \\
(1.1)\end{array}$ & $\begin{array}{c}1.1 \\
(1.1)\end{array}$ & 0.2 & $\begin{array}{c}1.1 \\
(1.1)\end{array}$ & $\begin{array}{c}1.1 \\
(1.1)\end{array}$ & 1.0 \\
\hline $\begin{array}{l}\text { Weighted aMMI, } \\
\text { mean (SD) }\end{array}$ & $\begin{array}{c}4.1 \\
(5.2)\end{array}$ & $\begin{array}{c}4.0 \\
(4.7)\end{array}$ & 0.9 & $\begin{array}{c}4.0 \\
(5.2)\end{array}$ & $\begin{array}{c}4.1 \\
(4.7)\end{array}$ & 0.3 & $\begin{array}{c}4.0 \\
(5.0)\end{array}$ & $\begin{array}{c}4.0 \\
(4.9)\end{array}$ & 1.0 \\
\hline
\end{tabular}

aMMI= adapted MultiMorbidity Index; $\mathrm{CDAl}=$ Clinical Disease Activity Index; SDAl= Simplified Disease Activity Index Proportion of patients achieving LDA between patients with binary $\mathrm{aMMl}=0$ and binary $\mathrm{aMMl}=1$. Because of no statistically significant results, no multivariate analysis was performed.
Table 2. Probability of first DMARD maintenance at 1, 3, 5 and 10 years (multivariate analysis)

\begin{tabular}{|c|c|c|c|c|}
\hline \multirow[t]{2}{*}{ Time point } & \multirow{2}{*}{$\begin{array}{l}\text { First DMARD maintained or } \\
\text { stopped }\end{array}$} & \multicolumn{2}{|c|}{$\begin{array}{l}\text { Binary } \\
\text { aMMI }^{\#}\end{array}$} & \multirow{2}{*}{$\begin{array}{l}\text { Counted } \\
\text { aMMI }^{\S}\end{array}$} \\
\hline & & 0 & 1 & \\
\hline \multirow[t]{2}{*}{1 year } & Maintenance $(n=300)$ & 2 & 298 & $1.71(0.93)$ \\
\hline & OR $[95 \% \mathrm{Cl}]^{*}$ & \multicolumn{2}{|c|}{$>999$} & $\begin{array}{c}221.3 \\
{[84.0-583.0]}\end{array}$ \\
\hline$(n=530)$ & Withdrawal $(n=230)$ & 205 & 25 & $0.12(0.37)$ \\
\hline 3 years & $\begin{array}{l}\text { Maintenance }(\mathrm{n}=285) \\
\quad \text { OR }[95 \% \mathrm{Cl}]^{*}\end{array}$ & 153.9 & $9^{275}$ & $\begin{array}{c}1.66(0.94) \\
26.1\end{array}$ \\
\hline \multirow[t]{2}{*}{$(n=493)$} & & \multicolumn{2}{|c|}{ [73.0-324.5] } & [15.1-45.3] \\
\hline & Withdrawal $(n=208)$ & 175 & 33 & $0.22(0.64)$ \\
\hline 5 years & $\begin{array}{c}\text { Maintenance }(n=116) \\
\text { OR }[95 \% \mathrm{Cl}]^{*}\end{array}$ & \multicolumn{2}{|c|}{10.9} & $\begin{array}{c}1.72(1.05) \\
2.2\end{array}$ \\
\hline \multirow[t]{2}{*}{$(n=459)$} & & \multicolumn{2}{|c|}{ [5.1-23.3] } & {$[1.8-2.7]$} \\
\hline & Withdrawal $(n=343)$ & 163 & 180 & $0.82(1.0)$ \\
\hline 10 years & $\begin{array}{l}\text { Maintenance }(n=40) \\
\text { OR }[95 \% \mathrm{Cl}]^{*}\end{array}$ & \multicolumn{2}{|c|}{14.0} & $\begin{array}{c}1.58(0.84) \\
1.6\end{array}$ \\
\hline \multirow[t]{2}{*}{$(n=415)$} & & \multicolumn{2}{|c|}{ [3.3-59.1] } & {$[1.2-2.0]$} \\
\hline & Withdrawal $(n=375)$ & 158 & 217 & $0.99(1.12)$ \\
\hline
\end{tabular}

"data are number of patients ${ }^{\S}$ data are mean (standard error)* data are odds ratios (ORs) and $95 \%$ confidence intervals $(95 \% \mathrm{Cl})$ of still being on the first initiated DMARD at 1, 3, 5 and 10 years between patients with binary $\mathrm{aMMI}=1$ and binary $\mathrm{aMMI}=0$ and according to counted aMMI, per additional point.

The variables included in multivariate analyses were sex, rheumatoid factor and/ or anti-citrunillated peptide antibody positivity, age, CDAl at baseline visit, number of treatments at baseline visit.

Acknowledgements: We are grateful to Nathalie Rincheval (Montpellier) for expert monitoring and data management and all the investigators who recruited and followed the patients (F. Berenbaum, Paris-Saint Antoine; MC. Boissier, Paris-Bobigny; A. Cantagrel, Toulouse; B. Combe, Montpellier; M. Dougados, Paris-Cochin; P. Fardellone and P. Boumier, Amiens; B. Fautrel, Paris-La Pitié; RM. Flipo, Lille; Ph. Goupille, Tours; F. Liote, Paris- Lariboisière; O. Vittecoq, Rouen X. Mariette, Paris-Bicêtre; P. Dieude, Paris Bichat; A. Saraux, Brest; T. Schaeverbeke, Bordeaux; and J. Sibilia, Strasbourg).

Disclosure of Interests: Aurélie BELTAI: None declared, Bernard Combe Speakers bureau: AbbVie; Bristol-Myers Squibb; Gilead; Janssen; Lilly; Merck; Novartis; Pfizer; Roche-Chugai; and Sanofi, Consultant of: AbbVie; Bristol-Myers Squibb; Gilead; Janssen; Lilly; Merck; Novartis; Pfizer; Roche-Chugai; and Sanofi, Grant/research support from: Novartis, Pfizer, and Roche, Amandine Coffy: None declared, Cécile Gaujoux-Viala: None declared, Cédric Lukas Speakers bureau: Abbvie, Amgen, Janssen, Lilly, MSD, Novartis, Pfizer, Roche-Chugai and UCB, Consultant of: Abbvie, Amgen, Janssen, Lilly, MSD, Novartis, Pfizer, RocheChugai and UCB, Grant/research support from: Pfizer, Novartis and RocheChugai, Alain Saraux Speakers bureau: AbbVie; Bristol-Myers Squibb; Lilly; Nordic; Novartis; Pfizer; Roche-Chugai; Sanofi and UCB, Consultant of: AbbVie; Bristol-Myers Squibb; Lilly; Nordic; Novartis; Pfizer; Roche-Chugai; Sanofi and UCB, Grant/research support from: AbbVie; Bristol-Myers Squibb; Lilly; Nordic; Novartis; Pfizer; Roche-Chugai; Sanofi and UCB, Maxime Dougados Speakers bureau: Pfizer, Abbvie, Lilly, UCB, Merck, BMS, Roche, Biogen, Sanofi, Novartis, Sandoz, Consultant of: Pfizer, Abbvie, Lilly, UCB, Merck, BMS, Roche, Biogen, Sanofi, Novartis, Sandoz, Grant/research support from: Pfizer, Abbvie, Lilly, UCB, Merck, BMS, Roche, Biogen, Sanofi, Novartis, Sandoz, Jean-Pierre DAURES: None declared, Charlotte Hua: None declared DOI: 10.1136/annrheumdis-2021-eular.2868

\section{\begin{tabular}{|l|l}
\hline POS0307 & FATIGUE IN RHEUMATOID ARTHRITIS PATIENTS IS
\end{tabular} ASSOCIATED TO SUBJECTIVE BUT NOT OBJECTIVE ASSESSMENTS OF DISEASE ACTIVITY DURING TREATMENT WITH TOCILIZUMAB}

H. B. Hammer ${ }^{1}$, B. Agular ${ }^{2}$, L. Terslev ${ }^{3} .{ }^{1}$ Diakonhjemmet Hospital,

Rheumatology, Oslo, Norway; ${ }^{2}$ Roche, Rheumatology, Copenhagen, Denmark;

${ }^{3}$ Rigshospitalet Glostrup, Rheumatology, Copenhagen, Denmark

Background: Fatigue is common in patients with rheumatoid arthritis (RA), and its association with inflammatory activity is not fully understood.

Objectives: To explore the associations between fatigue and inflammation by clinical, laboratory and ultrasound examinations during follow-up of RA patients on biological treatment. 